\title{
Women's Health Learning Package: a rich source of knowledge for global health providers
}

\author{
S. Chhabra
}

\section{Introduction}

The issues of development, environment and health are closely related. This reflects the complex link between the social, economic, ecological and political factors that determine the standard of living and other aspects of social well-being that influence human health. A healthy population and safe environment are important pre-conditions for a sustainable future and so healthy communities are essential (Fien et al., 2001; 2010).

Health professionals education and learning need to be as is required for the health of the communities. It is essential that health professionals are able to provide the best health care. They need to learn about ground realities of health issues, solutions and must find ways to help themselves. In this direction the Women and Health Taskforce of Network of Towards Unity For Health (TUFH) with the support of Global Health through Education Training and Service (GHETS) is developing a series of training modules based on prevalent issues affecting women's health. These modules can help professionals in learning about various aspects of women's health either by self- learning workshops or by focus group discussions. Some of the modules include:

1. Adolescent Health

2. Gender and Health

3. Cervical Cancer

4. Use of Medicines

5. Safe Motherhood

6. Men's Involvement in Promoting Reproductive Health

Director, Professor

Obstetrics \& Gynaecology,

Mahatma Gandhi Institute Of Medical Sciences,

Sevagram, Wardha - 442 102, Maharashtra, India

Email : chhabra_s@rediffmail.com
7. Nutrition and Women's Health

8. Menopause

9. Female Genital Mutilation

10. Mother-to-Child Transmission of HIVIAIDS

11. Domestic Violence

12. Post Partum Depression - Case Study

13. High Risk Pregnancy and Medical Decisions-Case Study

14. Treating Complications in Pregnancy Case Study

15. Unwanted Pregnancy and Unsafe Abortion

16. Violence Against Women

17. Contraceptive Practices

Modules have scope for change/alterations, be updated, so that health professionals learn the latest solutions of health problems. This can include birth to death as well as culture and environmental factors.

However, the concepts of updating and revising of the modules depends on needs with feedback from end users. These modules are part of the Women and Health Learning Package (WHLP), a freely accessible, e-learning resource available at www.GHETS.org used by educators, health providers and health sciences students (particularly medical and nursing students) around the world. These modules have relevant case studies with real life experiences by personnel around the world giving a stimulus for learning about the health of women around the globe essential for appropriate medical education.

The parent body of WHTF is Network Towards Unity for Health (TUFH), a non-governmental organization, which is a global association of individuals, groups, institutions and organizations which is the World Health Organization (WHO). WHO is committed to improving and maintaining health of communities and has a mandate to serve. The Headquarter of TUFH is in Ghent, Belgium, www.the-networktufh.org. Earlier this organization was called Network of Community Oriented Medical Institutes with its headquarters in Maastricht, Netherlands. 
Global Health through Education, Training (GHET), founded in 2002 by a small group of physicians and health advocates, many of whom were active members of The Network: Community Partnerships for Health through Innovative Education, Service, and Research, and has been renamed in 2003 as the Global Health Through Education, Training and Service (GHETS) to better reflect the organization's mission and priorities. It is another non-governmental, non-profit organization based in the USA, dedicated to improving health of all, especially in developing countries through innovations in education and service. GHETS is a seed funding and program-development partner of The Network: TUFH.

GHETS provides start-up grants to training institutions in low-income countries, and the technical help to launch and improve programs that prepare and support medical education and health care workers in rural and poor communities. GHETS believes that well trained and supported doctors, nurses and allied health workers are key to sustainable change. GHETS supports and enhances local infrastructure in developing countries, focusing on educational institutions to increase the number of trained medical professionals in the workforce by working with local program partners, GHETS programs are developed and completed on the ground by individuals who understand the small changes that are necessary to make large sustainable impacts on their communities. GHETS visualizes that one of the best and, most affordable ways to save most lives and improve overall health is to increase the number of trained local, primary healthcare workers. By leveraging small investments and the leadership of local change agents, GHETS' programs offer the most cost-effective and sustainable strategies to recruit, train and support primary care workers in developing countries, working with a network of medical and nursing schools in developing countries to provide comprehensive healthcare.

GHETS and The Network: TUFH have joined forces to promote strategies for change that address the health related challenges of global communities. WHLP has been developed just to do that. It will increase the ability of comprehensive care as there is access to real life knowledge. These accessible resource sharing provides training for the next generation of health workers, and provides information for evidence based clinical practice and stimulus for research worldwide.

\section{References:}

Fien, J., Spork, H. \& O'Donoghue, R. (2001) Teaching and Learning for a Sustainable Future[Online], (Updated in 2010 ) Available at: http://www.unesco.org 